\title{
FIBER PRODUCTS OF ELLIPTIC SURFACES WITH SECTION AND ASSOCIATED KUMMER FIBRATIONS.
}

\author{
GRZEGORZ KAPUSTKA AND MICHA£ KAPUSTKA
}

\begin{abstract}
We investigate Calabi-Yau three folds which are small resolutions of fiber products of elliptic surfaces with section admitting reduced fibers. We start by the classification of all fibers that can appear on such varieties. Then, we find formulas to compute the Hodge numbers of obtained three folds in terms of the types of singular fibers of the elliptic surfaces. Next we study Kummer fibrations associated to these fiber products.
\end{abstract}

\section{INTRODUCTION}

Calabi-Yau three folds which are small resolutions of nodal fiber products of relatively minimal rational elliptic surfaces with section were introduced by Schoen in [33. This class of manifolds appeared to be a very good background for studying for instance arithmetics and modularity. For instance, most of the examples of non rigid modular Calabi-Yau three folds are connected in some way with this family.

The main goal of this article is to extend the family studied by Schoen.

In the first part of the paper we investigate Calabi-Yau three folds which are small resolutions of fiber products of elliptic surfaces with section admitting reduced fibers. We allow all types and configurations of such fibers on the rational elliptic surfaces and hence obtain more complicated rational double points to resolve. We start by the classification of all fibers that can appear on such varieties. Then, we find formulas to compute the Hodge numbers of obtained three folds in terms of the types of singular fibers of the elliptic surfaces. Next we focus on finding geometric interpretation of the deformations of studied manifolds. In the general context (when the fibers of the elliptic surfaces can be nonreduced) we prove that a generic deformation of a Calabi-Yau resolution of a fiber product of two elliptic surfaces with section is birational to such a fiber product. In more special cases we give two ways of interpretation. The first is in terms of deformations of the configurations of singular fibers on both elliptic surfaces. The second bases on the description of studied three folds as iterated covers of $\mathbb{P}^{3}$ branched along two quartic cones.

The rest of the paper is devoted to the study of Kummer fibrations associated to these fiber products. Their construction consists of taking the quotient of a fiber product of two elliptic surfaces with section by a suitable involution and next resolving obtained singularities. The described three folds admit fibrations by Kummer surfaces. In general fibred manifolds play a special role in arithmetics. Moreover, general three folds fibred by K3 surfaces are still not well understood. The advantage of the Kummer fibrations constructed is that they belong to the

Mathematical subject classification 14J32; 14J27.

The project is co-financed by the EU funds and national budjet. 
well described family of double octics. This enables us to study the Hodge numbers of these varieties.

Acknowledgements We would like to thank our advisor Dr hab. S. Cynk for his constant enormous help during the work on this paper. We are grateful to Prof. A. Langer for his precious remarks. We would like also to thank the Referee of this paper for many interesting suggestions.

\section{The Fiber PRoduCtS OF ELLIPTIC SURFACES WITH SECTION}

In 33. Schoen introduced a new class of Calabi-Yau varieties. These are small resolutions of nodal fiber products of relatively minimal elliptic surfaces with section. This family was investigated by many algebraic geometers. In this section we would like to extend Schoen's construction and study obtained varieties a bit closer.

In this paper by the term elliptic surface we mean a surface $S$ together with a morphism $\pi: S \rightarrow \mathbb{P}^{1}$ such that the generic fiber of $\pi$ is a smooth elliptic curve. By abuse of notation we denote such elliptic surface simply by $S$. Using this term we moreover require that $S$ is minimal in the sense that it does not have -1 curves as components of fibers. An elliptic surface $S$ admits a section if the associated morphism $\pi$ admits a section. For simplicity we identify the section with its image.

Let $S_{1}$ and $S_{2}$ be two rational elliptic surfaces with section and assume that they have only reduced fibers. Let $X$ be the fiber product $S_{1} \times \mathbb{P}_{1} S_{2}$. If we see it as the inverse image of the diagonal in $\mathbb{P}^{1} \times \mathbb{P}^{1}$ to the product $S_{1} \times S_{2}$, using the adjunction formula (for more details see [33]) we prove that $X$ is a singular Calabi-Yau three fold. Hence, if $X$ admits a small resolution $\hat{X}$, then $\hat{X}$ is a Calabi-Yau three fold. In this section we are interested in studying Hodge numbers of such Calabi-Yau three folds.

Let us start with the following proposition.

Proposition 1.1. Let $S_{1}$ and $S_{2}$ be two rational elliptic surfaces with section. Then the three fold $X=S_{1} \times \mathbb{P}^{1} S_{2}$ admits a small resolution if and only if all fibers of $X$ are of one of the following types: $F \times I_{0}, I_{n} \times I_{m}, I I I \times I_{n}, I I I \times I I I, I V \times I_{n}$, $I I \times I I$, where $F$ denotes any fiber of an elliptic ruled surfaces.

Proof. Observe that all the singularities of $X$ are lying on a fiber which is the product of two singular fibers. We can find explicitly the local equations of the variety around each of these singularities and check which ones admit a small resolution.

The local equations of the surfaces around a singularity of the fiber are the following:

$I_{n}$ The local equation around each singular point of the fiber is $t=x y$.

II The local equation around the singularity of the fiber is $t=y^{2}-x^{3}$.

III The local equation around the singularity of the fiber is $t=x\left(y^{2}-x\right)$.

$I V$ The local equation around the singularity of the fiber is $t=x y(x+y)$.

In each of these cases the fibration is given by the projection onto the coordinate $t$. It follows that the fiber product around each of its singularities has one of the following local equations.

$I_{n} \times I_{m}$ The local equation around each of the $n \cdot m$ singularities is $x y=u^{2}+v^{2}$. This is an equation defining a node, which admits a small resolution. 
$I_{n} \times I I$ The local equation around each of the $n$ singularities is $x y=u^{2}-v^{3}$. This is an equation of an $A_{2}$ singularity. It does not admit a small resolution as it is factorial.

$I_{n} \times I I I$ The local equation around each of the $n$ singularities is $x y=u\left(v^{2}-u\right)$. This equation defines an $A_{3}$ singularity. It admits a small resolution which is given by the blowing up of the plane $\{x=u=0\}$.

$I_{n} \times I V$ The local equation around each of the $n$ singularities is $x y=u v(u+v)$. This is a singularity of type $D_{4}$. It admit a small resolution which is given by the consecutive blowing up of the plane $\{x=u=0\}$ and the proper transform of $\{y=v=0\}$.

$I I \times I I$ The local equation around the singularity is $x^{2}-y^{3}=u^{2}-v^{3}$. This is also a singularity $D_{4}$. Its small resolution is given by the blow up of the plane $x-u=y-v=0$ and then taking a small resolution of the resulting node.

$I I \times I I I$ The local equation around the singularity is $x^{2}-y^{3}=u\left(v^{2}-u\right)$. This is a singularity of type $E_{6}$, which is factorial from [20, thm B]. Hence it does not admit any small resolution.

$I I \times I V$ The local equation around the singularity is $x^{2}-y^{3}=u v(u+v)$. It is a double cover of $\mathbb{C}^{3}$ ramified over a triple point. From [20, thm B] it is a factorial singularity. It does not admit any small resolution.

$I I I \times I I I$ The local equation around the singularity is $x\left(y^{2}-x\right)=u\left(v^{2}-u\right)$. It admits a small resolution which is given by the blowing up of the plane $x=u=0$ and a small resolution of resulting nodes.

$I I I \times I V$ The local equation around the singularity is $x\left(y^{2}-x\right)=u v(u+v)$. This is a singularity that does not admit any small resolution. We can see this in the following way. After blowing up the plane $x=u=0$ we obtain an $A_{2}$ hence factorial singularity. Now we can use an argument of Kollár to prove that the small resolution does not exist. Indeed, if we had such a resolution, then it would differ from the obtained $A_{2}$ by a sequence of flops [17, theorem 4.9]. This gives a contradiction with the fact that flops in dimension 3 do not change the types of singularities [17, theorem 2.4].

$I V \times I V$ The local equation around the singularity is of type $x y(x+y)=u v(u+v)$. This is a simple triple point. It admits a big crepant resolution, hence do not have any small one.

Proposition 1.2. The three fold $X$ admits a projective small resolution $\hat{X}$ if moreover no fiber is of type $I I \times I I, I I I \times I I I$ or $I_{1} \times F$, where $F$ is a singular fiber.

Proof. We easily check that for each of allowed singularities the analytical small resolution described above is given by a sequence of blowings up of components of fibers (they are all isomorphic to $\mathbb{P}^{1} \times \mathbb{P}^{1}$ ).

To study Hodge numbers of constructed varieties we introduce some notation. Let $A_{1}, A_{2} \subset \mathbb{P}^{1}$ be the sets of singular fibers of surfaces $S_{1}$ and $S_{2}$ respectively. Let $S^{\prime \prime}=A_{1} \cap A_{2}$. Let $b_{t}$ and $b_{t}^{\prime}$ for $t \in \mathbb{P}^{1}$ denote the number of components of the fibers over the point $t$ of $S_{1}$ and $S_{2}$ respectively.

Let us start by computing $h^{1,1}(\hat{X})$. We adapt the arguments of 33 to our wider context. We have the exact sequence

The free abelian group generated by all components of the fibers

$\longrightarrow \operatorname{Pic}(X) \longrightarrow \operatorname{Pic}\left(X_{\eta}\right) \longrightarrow 0$ 
where $X_{\eta}$ denotes the generic fiber of $X$ in the sense of schemes. Moreover, all relations between images of components of the fibers are induced by the linear equivalence of the fibers. We get

$$
\operatorname{rk} \operatorname{Pic}(\hat{X})=1+\sum_{t \in A_{1} \cup A_{2}}\left(b_{t} b_{t}^{\prime}-1\right)+\operatorname{rk} \operatorname{Pic}\left(\hat{X}_{\eta}\right) .
$$

Continuing, define $d(X)=1$ if and only if $S_{1}$ and $S_{2}$ are isogenous and $d(X)=0$ otherwise. Let $S_{1 \eta}, S_{2 \eta}$ denote the generic (in the sense of schemes) fibers of the respective surfaces $S_{1}$ and $S_{2}$

$\operatorname{rk} \operatorname{Pic}\left(\hat{X}_{\eta}\right)=\operatorname{rk} \operatorname{Pic}\left(S_{1 \eta}\right)+\operatorname{rk} \operatorname{Pic}\left(S_{2 \eta}\right)+d(X)=18-\sum_{t \in A_{1}}\left(b_{t}-1\right)-\sum_{t \in A_{2}}\left(b_{t}^{\prime}-1\right)+d(X)$.

Together this gives

$$
h^{1,1}(\hat{X})=\sum_{t \in A_{1} \cup A_{2}}\left(b_{t} b_{t}^{\prime}-1\right)+19-\sum_{t \in A_{1}}\left(b_{t}-1\right)-\sum_{t \in A_{2}}\left(b_{t}^{\prime}-1\right)+d(X) .
$$

Now, to get a formula for $h^{1,2}(\hat{X})$ we compute the Euler characteristic of $\hat{X}$. First for $t \in \mathbb{P}^{1}$ define $F_{t}$ and $F_{t}^{\prime}$ to be the fibers over $t$ of surfaces $S_{1}$ and $S_{2}$ respectively. For all $s \in \operatorname{Sing}(X)$ define $E_{s}$ to be the exceptional curve of the small resolution of the singularity in $s$. Then

$$
\chi(\hat{X})=\chi(X)+\sum_{s \in \operatorname{Sing}(X)}\left(\chi\left(E_{s}\right)-1\right)
$$

and

$$
\chi(X)=\sum_{t \in S^{\prime \prime}} \chi\left(F_{t}\right) \chi\left(F_{t}^{\prime}\right) .
$$

Hence, to compute the Euler characteristic $\chi(\hat{X})$ we need only to compute the Euler characteristics of the fibers of the surfaces, and of the exceptional curves of each resolved singularity. We already have

$$
\chi(F)=\left\{\begin{array}{l}
n \text { for } F \text { of type } I_{n} \\
3 \text { for } F \text { of type } I I I \\
4 \text { for } F \text { of type } I V .
\end{array}\right.
$$

To compute the Euler characteristic of an exceptional curve we only need to know the number of local blowings up of Weil divisors we performed during the resolution of singularities described in the proof of Proposition 1.1. This gives

$$
\chi\left(E_{i}\right)=\left\{\begin{array}{l}
2 \text { for all nodes and the singularities of } I_{n} \times I I I \\
3 \text { for the singularities of } I V \times I_{n}
\end{array}\right.
$$

Together this simplifies to a formula for the Euler characteristic depending only on the numbers of components of fibers

$$
\chi(\hat{X})=2 \sum_{t \in S^{\prime \prime}} b_{t} b_{t}^{\prime}
$$

To get the formula for $h^{1,2}$ we use

$$
h^{1,2}(\hat{X})=h^{1,1}(\hat{X})-\frac{\chi(\hat{X})}{2} .
$$


Finally we obtain

$$
h^{1,2}(\hat{X})=19+d(X)-\sum_{t \in S^{\prime \prime}}\left(b_{t}+b_{t}^{\prime}-1\right) .
$$

Remark 1.3. We have computed Hodge numbers for the class of projective CalabiYau three folds described in Proposition 1.2, Let us remind that any, including the non-projective, introduced Calabi-Yau small resolution is a Moishezon manifold. We can hence study its Hodge numbers. Moreover, in our case we can extend the above computations to all Calabi-Yau three folds $\hat{X}$. The only difference is that we need to put appropriate Euler characteristics of the exceptional divisors corresponding to obtained singularities on $X$. This is not hard as these depend only on the number of local blowings up in the resolutions described by the proof of Proposition 1.1

On any smooth Calabi-Yau three fold $X$ the number $h^{1,2}(X)$ is equal to the space of infinitesimal and by the theorem of Bogomolov-Tian-Todorov also small deformations of this three fold. Let us focus on this interpretation and find a natural local description of the Kuranishi space of $\hat{X}$.

We shall first present some argument concerning deformations of general CalabiYau three folds which are resolutions of fiber products of elliptic surfaces with section.

1.1. Deformations in the general case. In this subsection we do not restrict the type of fibers of the product and prove the following

Theorem 1.4. Let $X$ be a Calabi-Yau three fold, which is a resolution of a fiber product of two rational elliptic surfaces with section. Then a generic deformation of $X$ is birational to a fiber product of this type.

The proof is based on the following lemma.

Lemma 1.5. Let $X$ be a Calabi-Yau manifold containing the product of two elliptic curves $E_{1}$ and $E_{2}$. Assume moreover that $h^{0}\left(E_{1}, N_{E_{1} \mid X}\right)=h^{0}\left(E_{2}, N_{E_{2} \mid X}\right)=2$. Then $X$ is birational to a fiber product of rational elliptic surfaces with section.

Proof of Lemma 1.5. Let $A=E_{1} \times E_{2}$, where $E_{1}$ and $E_{2}$ are elliptic curves, be the abelian surface contained in $X$. We can easily compute that the linear system $|A|$ is one dimensional and next we obtain $X$ to admit an abelian fibration over $\mathbb{P}^{1}$ with special fiber $A$ (For more details see [11]).

The infinitesimal deformations of $A$ in $X$ are induced by the morphism

$$
H^{0}\left(A, N_{A \mid X}\right) \longrightarrow H^{1}\left(A, T_{A}\right)
$$

coming from the exact sequence

$$
\left.0 \longrightarrow T_{A} \longrightarrow T_{X}\right|_{A} \longrightarrow N_{A \mid X} \longrightarrow 0 .
$$

We will prove that these deformations of $A$ in $X$ induce deformations of pairs $\left(A, E_{1}\right)$ and $\left(A, E_{2}\right)$. Consequently a generic abelian fiber of the fibration will contain two elliptic curves intersecting in one point, thus will be a product.

Let us concentrate on the deformation of $\left(A, E_{1}\right)$. We have two exact sequences

$$
\begin{gathered}
\left.0 \longrightarrow T_{E_{1}} \longrightarrow T_{A}\right|_{E_{1}} \longrightarrow N_{E_{1} \mid A} \longrightarrow 0 \\
\left.0 \longrightarrow T_{A}\left(-E_{1}\right) \longrightarrow T_{A} \longrightarrow T_{A}\right|_{E_{1}} \longrightarrow 0
\end{gathered}
$$


Together with the exact sequence 1.1 they induce a diagram in cohomology.

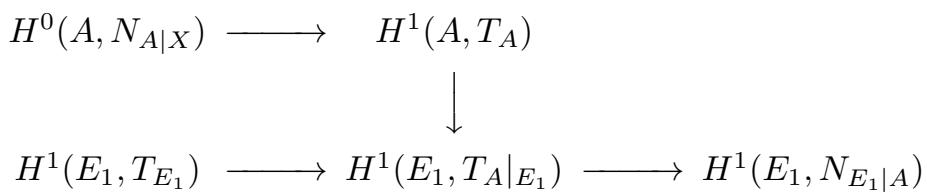

We need to prove that there are no obstructions to lift the considered infinitesimal deformations of $A$ in $X$ to deformations of $E_{1}$ in $X$, i.e. that the composition morphism

$$
H^{0}\left(A, N_{A \mid X}\right) \longrightarrow H^{1}\left(A, T_{A}\right) \longrightarrow H^{1}\left(E_{1},\left.T_{A}\right|_{E_{1}}\right) \longrightarrow H^{1}\left(E_{1}, N_{E_{1} \mid A}\right),
$$

is trivial. Observe that this morphism factorizes by the suitable morphism induced by the following exact sequence:

$$
\left.0 \longrightarrow N_{E_{1} \mid A} \longrightarrow N_{E_{1} \mid X} \longrightarrow N_{A \mid X}\right|_{E_{1}} \longrightarrow 0
$$

From the adjunction formula $\left.N_{E_{1} \mid A} \simeq N_{A \mid X}\right|_{E_{1}} \simeq \mathcal{O}_{E_{1}}$. Now from the assumption and the associated cohomology sequence

$0 \longrightarrow H^{0}\left(E_{1}, N_{E_{1} \mid A}\right) \longrightarrow H^{0}\left(E_{1}, N_{E_{1} \mid X}\right) \longrightarrow H^{0}\left(E_{1},\left.N_{A \mid X}\right|_{E_{1}}\right) \longrightarrow H^{1}\left(E_{1}, N_{E_{1} \mid A}\right)$

for dimension reasons the morphism $H^{0}\left(E_{1}, N_{E_{1} \mid X}\right) \longrightarrow H^{0}\left(E_{1},\left.N_{A \mid X}\right|_{E_{1}}\right)$ is surjective. Hence $H^{0}\left(E_{1},\left.N_{A \mid X}\right|_{E_{1}}\right) \longrightarrow H^{1}\left(E_{1}, N_{E_{1} \mid A}\right)$ is trivial.

To finish the proof we remind that deformations of abelian surfaces on $X$ as well as deformations of elliptic curves are unobstructed. Hence the obtained deformations of elliptic curves $E_{1}$ and $E_{2}$ sweep out two elliptic surfaces $S_{1}$ and $S_{2}$. The three fold $X$ is then birational to the fiber product $S_{1} \times_{\mathbb{P}_{1}} S_{2}$. The two intersecting elliptic curves in each smooth fiber yield sections in both elliptic surfaces. Moreover, as the deformation of a Calabi-Yau three fold is a Calabi-Yau three fold the two surfaces $S_{1}$ and $S_{2}$ are rational (see [33]).

We are now ready to prove the main theorem:

Proof of Theorem 1.4. Let $X$ be a resolution of singularities of a fiber product $Y$ of two rational elliptic surfaces with section. Then $X$ contains a surface $A$ which is a product of two elliptic curves. By [37] the Kähler cone of $X$ and the Kähler cone of a generic deformation $X^{\prime}$ of $X$ can be seen as subcones of the same space $H^{2}(X, \mathbb{R})$. From the same paper we know that each abelian fibration is given by a divisor $D_{A}$ such that $D_{A}^{3}=0, D_{A}^{2} \equiv 0, c_{2}(X) \cdot D_{A}=0$ and each elliptic fibration is given by $D_{E}$ such that $D_{A}^{3}=0, D_{A}^{2} \not \equiv 0, c_{2}(X) \cdot D_{A} \geq 0$. Moreover by [40] the Kahler cone of $X$ is a subcone of the Kahler cone of $X^{\prime}$ cut out by some half space. By [42, cor. 2, rem. 1] the divisors $D_{E_{1}}$ and $D_{E_{2}}$ induce also two elliptic fibrations on $X^{\prime}$.

We are interested in finding an abelian surface containing two elliptic curves each from a different fibration. Let $S$ be a section of the fibration given by $D_{E_{1}}$ on $X$ (such section exists as $X$ is a fiber product of two elliptic surfaces with section). Then $S$ is a rational elliptic surface. In particular $H^{1}\left(S, \mathcal{O}_{S}\right)=0$, hence any deformation $X^{\prime}$ of $X$ induces a deformation $S^{\prime}$ of $S$. As the intersection form is fixed in the deformation, $S^{\prime}$ is covered by fibers of the fibration given by $D_{E_{1}}$ and cuts the fibers of the fibration given by $D_{E_{2}}$ in one point. Thus we can consider the surface $A^{\prime}$ on $X^{\prime}$ swept out by curves from one fibration over a curve from 
the second. Then $A^{\prime 2} \equiv 0, A^{3}=0$ and $A^{\prime}$ is not a K3 surface (it admits an elliptic fibration over an elliptic curve). This implies that $A$ is an abelian surface on $X^{\prime}$ containing two elliptic curves $E_{1}^{\prime}, E_{2}^{\prime}$ meeting in one point. Hence it is the product of these two curves. Moreover as both curves come from two-dimensional fibrations we have $h^{0}\left(E_{1}^{\prime}, N_{E_{1}^{\prime} \mid X^{\prime}}\right)=h^{0}\left(E_{2}^{\prime}, N_{E_{2}^{\prime} \mid X^{\prime}}\right) \geq 2$. By lemma 1.5 this ends the proof.

Let us moreover point out the following

Remark 1.6. If a ruled surface over a curve of genus $g \geq 1$ is contained in $X$, then it has to be a component of a fiber of type $I_{0} \times F$.

Indeed suppose to the contrary. Let $S$ be such a surface contained in $X$. Then there exists a surjection from $S$ to one of the elliptic surfaces (as the fiber product of two multi-sections is a multi-section). The inverse-image $F$ of the generic elliptic fiber have to be an effective smooth (as it is base point free) divisor with selfintersection 0 . Hence one of the following holds:

- The divisor $F$ is the sum of some fibers of the ruled surface. This is impossible as there exists no morphism from $\mathbb{P}^{1}$ to a curve of positive genus (Lüroth's theorem).

- The surface $S$ is a product of a genus $g$ curve with a line. This is impossible since the inverse image of the section of the elliptic surface would have to be an exceptional curve. Such curves do not exist on products.

Corollary 1.7. A generic Calabi-Yau manifold from the Kuranishi space of a fiber product of two rational elliptic surfaces with section is also a fiber product of two rational elliptic surfaces with section and has no fibers of type $I_{0} \times F$ for $F \neq I_{1}, I I$

1.2. Deformations of constructed three folds. In this subsection we study projective Calabi-Yau three folds $X$ introduced in Proposition 1.2. We are looking for a more precise description of the space of small deformations of $\hat{X}$. That is we would like to see deformations from the point of view of singular fibers. Let us first find a generic variety obtained as a deformation of $\hat{X}$.

Proposition 1.8. A generic small deformation $\hat{Y}$ of $\hat{X}$ is a small resolution of a fiber product $Y$ of two elliptic surfaces $R_{1}$ and $R_{2}$ which are small deformations of $S_{1}$ and $S_{2}$ respectively and such that each fiber of $Y$ corresponds to a fiber of $X$ with the same number of components. Moreover, such variety $Y$ has no fibers of type $F \times I_{0}$ for $F \neq I_{1}$ or $I I$.

Proof. Let us consider the Weierstrass type equations of the surfaces $S_{1}=\left\{y^{2}=\right.$ $\left.x^{3}+f(t) x+g(t)\right\}$ and $S_{2}=\left\{y^{\prime 2}=x^{\prime 3}+f^{\prime}(t) x^{\prime}+g^{\prime}(t)\right\}$, where $f, g, f^{\prime}, g^{\prime}$ are polynomials of degrees 4,6,4,6 respectively. Consider the set $Q$ of all quadruples of polynomials $F, G, F^{\prime}, G^{\prime}$, of degrees 4,6,4,6 respectively, satisfying the following conditions

(1) The pairs $F, G$ and $F^{\prime}, G^{\prime}$ define by the Weierstrass type equations two elliptic surfaces $R_{1}$ and $R_{2}$.

(2) There is a bijection $\beta$ between the set $R^{\prime \prime}$ of common singular fibers of $R_{1}$ and $R_{2}$ and the corresponding set $S^{\prime \prime}$.

(3) for all $t \in R^{\prime \prime}$ the number of components of the fibers over $t$ of both surfaces $R_{1}$ and $R_{2}$ are the same as the numbers of components of fibers of respective surfaces $S_{1}$ and $S_{2}$ over $\beta(t)$. 
Claim The set $Q$ is an algebraic set of dimension at least $h^{1,2}(\hat{X})+5$.

The set is clearly nonempty. Hence to compute its dimension we compute the number of conditions we impose on the set of all quadruples of polynomials of respective degrees $4,6,4,6$. Note that requiring that a pair $(F, G)$ defines a surface with a fiber with $b_{t}$ components over a chosen $t$ is imposing $b_{t}$ conditions. Now:

- In the case where the surfaces $S_{1}$ and $S_{2}$ are not isogenous the number of conditions is $\sum_{t \in R^{\prime \prime}}\left(b_{t}+b_{t}^{\prime}-1\right)$.

- In the case where the surfaces $S_{1}$ and $S_{2}$ are isogenous the number of conditions describing isogenous pairs of surfaces is $\sum_{t \in R^{\prime \prime}}\left(b_{t}+b_{t}^{\prime}-1\right)-1$.

The claim is proved.

Element from the set $Q$ correspond to elliptic surfaces with fibers having the same number of components over $\beta(t)$ as the original surface in $t$. Let us consider the component of $Q$ containing the quadruple describing $X$. Using the fact that an elliptic rational surface has only one Weierstrass representation up to proportion and after dividing by the automorphism group of $\mathbb{P}^{1}$ we obtain a space of deformations of $X$ of dimension $h^{1,2}$.

To find a space of deformations for $\hat{X}$ we perform a simultaneous resolution of singularities in the family by blowing up the families of components of singular fibers.

We have found a space of dimension $h^{1,2}(\hat{X})$ describing deformations of $\hat{X}$. It has to be an open set in the Kuranishi space as the latter is smooth. By Corollary 1.7 the generic element of this set has no fibers of type $F \times I_{0}$ for $F \neq I_{1}$ or $I I$. This ends the proof.

From the above proof we can find a geometric description of deformations of $\hat{X}$ looking only at the singular fibers of $S_{1}$ and $S_{2}$ and their incidence conditions. Indeed each fiber of type $F \times I_{0}$ can be deformed in the deformation family into $\chi(F)$ fibers of type $I_{1} \times I_{0}$. A singular fiber of type $I_{n} \times F$ can be deformed into the sum of one singular fiber of type $I_{n} \times I_{m}$ with the same number of components as the original singular fiber and a fiber of type $I_{1} \times I_{0}$. More precisely:

- Fibers of type $I V \times I_{n}$ can deform into a fiber of type $I_{3} \times I_{n}$ and a fiber of type $I_{1} \times I_{0}$.

- Fibers of type $I I I \times I_{n}$ can deform into a fiber of types $I_{2} \times I_{n}$ and a fiber of type $I_{1} \times I_{0}$.

Moreover, fibers of type $I_{n} \times I_{m}$ have to preserve their type during the deformation.

Example 1.9. To ilustrate the result of the above theorem let us take two surfaces $S_{1}$ and $S_{2}$ given by Weierstrass equations as in the proof of above Lemma with:

- $f=12\left(t^{4}-t^{2}+1\right)$

- $g=4\left(2 t^{6}-3 t^{4}-3 t^{2}+2\right)$

- $f^{\prime}=3(t-3)^{2}(t-1)^{2}$

- $g^{\prime}=(t-3)^{2}(t-1)^{2}\left((t-2)^{2}+1\right)$

The discriminants of the surfaces are

- $\Delta=2^{4} 3^{6} t^{4}(t+1)^{2}(t-1)^{2}$,

- $\Delta^{\prime}=-108(t-3)^{4}(t-1)^{4}(t-2)^{2}$

In this case $S_{1}$ has singular fibers of types $I_{2} I_{2} I_{4} I_{4}$ in the points $-1,1,0, \infty$ and $S_{2}$ singular fibers of types $I_{2} I_{2} I V I V$ in the points $2, \infty, 3,1$. Their fiber product 
has one fiber of type $I_{2} \times I V$ and one fiber of type $I_{4} \times I_{2}$. Remaining fibers are of types $F \times I_{0}$. As described in the the proof of above Lemma the conditions imposed on quadruples $F, F^{\prime}, G, G^{\prime}$ describing the set $Q$ come from the requirement that the discriminants of both Weierstrass equations have two common zeroes of the following types. One of the common zeroes needs to be double for the frist discrminant and triple for the second and the second zero needs to be quadruple for the first discriminant and double for the second. These are 5+6-2 conditions on the space of discriminants hence also on $Q$. This gives $Q$ of dimension 15 . Finally the deformation space is of dimension 10 .

Remark 1.10. We did not prove that a fiber $F \times I_{n}$, where $F$ is an unstable fiber always splits in the deformation space. Using [26] we can prove that it is always possible to split a fiber of types $I I$ (resp. $I I I, I V$ ) into a sum of fibers $I_{1}$ and $I_{1}$ (resp. $I_{2}$ and $I_{1} ; I_{3}$ and $I_{1}$ ). However it is not clear that we can do it controlling the position of fibers.

Remark 1.11. In all above we did not need to assume that all fibers of $S_{1}$ and $S_{2}$ are reduced. We can allow fibers of type $F \times I_{0}$ for any $F$. Such fiber will also split in the deformation space into fibers of type $I_{1} \times I_{0}$.

1.3. The fiber product as an iterated double covering. Here, for a given fiber product of two elliptic surfaces with section we give another smooth model. This enables us to find an argument to compute the deformation space of $\hat{X}$ independently of the characteristic of the base field. Let us start with the following lemma concerning elliptic surfaces.

Lemma 1.12. Let $S$ be a rational elliptic surface with section. Assume moreover that all fibers of $S$ are reduced. Then $S$ is birational to the double covering $\hat{S}$ of $\mathbb{P}^{2}$ branched along a quartic curve $Q$ with only $A_{k}$ singularities. Moreover, we can choose this birational equivalence to map fibers of $S$ into the inverse images by the covering of lines passing through a fixed point not lying on $Q$.

Proof. Let us choose two disjoint sections $s_{1}$ and $s_{2}$ of $S$. To do this we use the fact that $S$ is the blow up of $\mathbb{P}^{2}$ in the base locus of a system of cubics (see [23]). By the assumption that all fibers are reduced we know that this system has at least two non-infinitely near base points. Then each of the at least two -1 exceptional divisors of the blowing up of the base set is a section and they are all pairwise disjoint.

Define an involution $i$ acting on fibers of $S$ such that $i\left(s_{1}\right)=s_{2}$. This can be done in the following way, we define $s_{1}$ to be the 0 section and take $i(x)=s_{2}-x$ on each fiber. The quotient of $S$ by $i$ is a ruled surface with section $s$ (the image of $s_{1}$ and $s_{2}$ ). After blowing down all components of the fibers that are disjoint from $s$ we obtain a minimal ruled surface $R$ with a -1 section and no section with self intersection less than -2 . But on the Hirzebruch surface $\mathbb{F}_{2}$ we do not have any -1 curves. Hence $R$ is the Hirzebruch surface $\mathbb{F}_{1}$ and $S$ is birational to the double cover of this $\mathbb{F}_{1}$ branched in a four-section disjoint from the section $s$. Blowing down the section $s$ we obtain the assertion of the Lemma. Indeed all singularities of the quartic obtained in this way are of type $A_{k}$ as resolving triple points of the branch locus lead to non-reduced fibers.

Remark 1.13. In fact the only elliptic surfaces with no two disjoint sections are the two surfaces $X_{22}$ and $X_{211}$ from [23] (the only rational elliptic surfaces with a fiber 
of type $I_{2}^{*}$ ). Hence we can generalize this lemma to all rational elliptic surface with section except the two above ones, we need only to allow all ADE singularities.

Remark 1.14. The above lemma and remark lead us to an interesting correspondence between elliptic surfaces with section (except $X_{22}$ and $X_{211}$ ) and Gorenstein del Pezzo surfaces of degree 2 (see Chapter 4).

For clarity let us consider the case when $S_{1}$ and $S_{2}$ have only semi-stable fibers. Let $\hat{S}_{1}$ and $\hat{S}_{2}$ be surfaces defined in Lemma 1.12. The fiber product $S_{1} \times_{\mathbb{P}^{1}} S_{2}$ is birational to the variety given in the product $\mathbb{P}(1,1,1,2) \times \mathbb{P}(1,1,1,2)$ with coordinates $(a, b, c, d ; \alpha, \beta, \gamma, \delta)$ by the equations

$$
d^{2}=Q_{1}(a, b, c), \quad \delta^{2}=Q_{2}(\alpha, \beta, \gamma), \quad a=\alpha, \quad b=\beta .
$$

This is birational to the variety $Y$ constructed as follows. Take the double cover of $\mathbb{P}^{3}$ branched over a quartic cone $Q_{1}(x, y, z)=0$ and next the double cover of the obtained variety branched over the inverse image of the cone $Q_{2}(x, y, t)=0$ by the first covering. Thus the fiber product $S_{1} \times_{\mathbb{P}^{1}} S_{2}$ is birational to the iterated double cover $Y$. Observe that $Y$ is a singular Calabi-Yau three fold with a smooth model $\hat{Y}$ constructed in the following way. We blow up $\mathbb{P}^{3}$ in such a way that the quartic cone $Q_{1}(x, y, z)=0$ is resolved, i.e. first we blow up the vertex of the cone then consecutively all the double lines (also the infinitely near). Next we take the first double covering branched over the proper transform of the cone. After that we do the same with the inverse image of the cone $Q_{2}(x, y, t)=0$ to the double cover. Here after blowing up the fourfold points and the double curves we obtain two new kind of singularities:

- Some nodes of the three fold lying outside the branch locus.

- Some nodes of the branch locus.

Both induce nodes on the double covering that we resolve by taking a small resolution.

The results of [7] give another method to compute the dimension $h^{1,2}(\hat{X})=$ $h^{1,2}(\hat{Y})$ of the space of deformations of $\hat{X}$.

Let us use the method in our context. We prove the following theorem

Theorem 1.15. Let $Y$ be an iterated double cover of $\mathbb{P}^{3}$ branched over two quartic cones $Q_{1}$ and $Q_{2}$ defining elliptic surfaces with semi-stable fibers. Assume moreover that $Q_{1}$ ad $Q_{2}$ do not pass through each other vertices. Then deformations of the small resolution of $Y$ correspond to those deformations of both cones that preserve the tangency relations and the multiplicities of the intersection points.

Example 1.16. Let us consider two reducible quartic cones in $\mathbb{P}^{3}$ (with coordinates $(x, y, z, t))$, given by equations $Q_{1}=\left\{x(x+z)\left((x-z)^{2}+t^{2}-25 z^{2}\right)=0\right\}$ and $Q_{2}=\left\{y(y+z)\left((x-3 z)^{2}+t^{2}-25 z^{2}\right)=0\right\}$. These two cones are tangent in the points $(1,3,1,5)$ and $(1,3,1,-5)$. They moreover intersect each other nontranversely in the point $(0,0,0,1)$. It is worth pointing out that the remaining points of intersection including the points of intersections of double lines on one cone with the other cone are treated as transversal, hence of multiplicity one. The above theorem says that deformations of a small resolution $\tilde{Y}$ of $\mathbb{P}^{3}$ branched over the two cones $Q_{1}$ and $Q_{2}$ correspond to such deformations of both cones that preserve tangency in one point and one quadruple point of intersection. More explicitly a small deformation of $\tilde{Y}$ is an iterated cover of $\mathbb{P}^{3}$ branched over two quartic cones tangent in one point and admitting double lines intersecting in one point. 
Corollary 1.17. Any deformation of a fiber product of two elliptic surfaces with section admitting only semi-stable fibers is a fiber product of elliptic surfaces with section with corresponding singular fibers consisting of the same number of components as in the deformed variety.

Proof. We have already proved that a fiber product of elliptic surfaces with section is a small resolution of an iterated double cover of $\mathbb{P}^{3}$ branched over two quartic cones. By Theorem 1.15 deformations of such varieties correspond to deformations of the cones. The deformed cones induce new fiber products. The only thing we need to observe is that a fiber of type $I_{n} \times I_{m}$ arise only in the case when the cones are either tangent to each other, or a double line of one cone is tangent to the other, or two double lines of the cones intersect. From the latter and Theorem 1.15 it follows that the fibers of type $I_{n} \times I_{m}$ are preserved in deformations, which is the assertion of the corollary.

Proof of theorem 1.15. Let us start with the observation that the space of deformations of a Calabi-Yau three fold which is a small resolution of a singular Calabi-Yau three fold $Y$ is isomorphic to the space of deformations of the variety which is a big resolution of $Y$. We study deformations of the three fold obtained by taking the big resolutions of the nodes.

We introduce the following notation:

- Let $D_{1}$ and $D_{2}$ denote the quartic cones from the theorem.

- Let $\sigma_{1}: \tilde{\mathbb{P}}^{3} \rightarrow \mathbb{P}^{3}$ be a sequence of blowups of $\mathbb{P}^{3}$ inducing a minimal resolution of $D_{1}$. The proper transforms of $D_{1}$ and $D_{2}$ by $\sigma_{1}$ will be denoted by $\tilde{D}_{1}$ and $\tilde{D}_{2}$. The line bundles corresponding to $\frac{1}{2} \tilde{D}_{1}$ and $\frac{1}{2} \tilde{D}_{2}$ will be denoted $\tilde{L}_{1}$ and $\tilde{L}_{2}$.

- Let $\varphi_{1}: \tilde{Z} \rightarrow \tilde{\mathbb{P}}^{3}$ be the double cover of $\tilde{\mathbb{P}}^{3}$ branched over $\tilde{D}_{1}$ and given by the corresponding line bundle $\tilde{L_{1}}$. The pullback of the bundle $\tilde{L}_{2}$ will be denoted by $\tilde{\mathcal{L}}_{2}$.

- Let $\sigma_{2}: \hat{Z} \rightarrow \tilde{Z}$ be a sequence of blow ups of $\tilde{Z}$ inducing a minimal resolution of $\varphi_{1}^{-1}\left(\tilde{D}_{2}\right)$. The proper transforms of $\varphi_{1}^{-1}\left(\tilde{D}_{1}\right)$ and $\varphi_{1}^{-1}\left(\tilde{D}_{2}\right)$ by $\sigma_{2}$ will be denoted by $\hat{D}_{1}$ and $\hat{D}_{2}$. The line bundle corresponding to $\frac{1}{2} \hat{D}_{2}$ will be denoted by $\hat{\mathcal{L}}_{2}$.

- Let $\varphi_{2}: \hat{Y} \rightarrow \hat{Z}$ be the double cover of $\hat{Z}$ branched over $\hat{D}_{2}$.

We have the following diagram

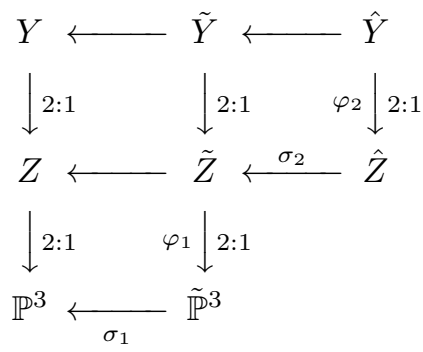

We are interested in computing $h^{1}\left(\hat{Y}, \Theta_{\hat{Y}}\right)$. As $\varphi_{2}$ is a finite morphism we have

$$
h^{1}\left(\hat{Y}, \Theta_{\hat{Y}}\right)=h^{1}\left(\hat{Z}, \varphi_{2 *}\left(\Theta_{\hat{Y}}\right)\right)=h^{1}\left(\hat{Z}, \Theta_{\hat{Z}} \otimes \hat{\mathcal{L}}_{2}^{-1}\right)+h^{1}\left(\hat{Z}, \Theta_{\hat{Z}}\left(\log \hat{D}_{2}\right)\right) .
$$


The first component of the sum can be computed using the fact that $\hat{Y}$ is a big resolution of a nodal Calabi-Yau three fold. We have then

$$
h^{1}\left(\hat{Z}, \Theta_{\hat{Z}} \otimes \hat{\mathcal{L}}_{2}^{-1}\right)=h^{1}\left(\tilde{Z}, \Theta_{\tilde{Z}} \otimes \tilde{\mathcal{L}}_{2}^{-1}\right)+\sum g\left(\hat{C}_{i}\right),
$$

where $g\left(\hat{C}_{i}\right)$ are the genera of the blown up curves. Each of these curves is a component of the pre-image of some double curve $C_{i}$ on the cone $D_{2}$. Hence their genera are equal to one if the curve $C_{i}$ was not tangent to the cone $D_{1}$ and zero otherwise. Taking again the direct image, this time by $\varphi_{1}$ we obtain by the projection formula

$$
h^{1}\left(\tilde{Z}, \Theta_{\tilde{Z}} \otimes \tilde{\mathcal{L}}_{2}^{-1}\right)=h^{1}\left(\tilde{\mathbb{P}}^{3}, \Theta_{\tilde{\mathbb{P}}^{3}} \otimes \tilde{L}_{1}^{-1} \otimes \tilde{L}_{2}^{-1}\right)+h^{1}\left(\Theta_{\tilde{\mathbb{P}}^{3}}\left(\log \tilde{D}_{1}\right) \otimes \tilde{L}_{2}^{-1}\right)=0
$$

We need to prove the last equality.

Proposition 1.18. The following equalities hold.

- $h^{1}\left(\tilde{\mathbb{P}}^{3}, \Theta_{\tilde{\mathbb{P}}^{3}} \otimes \tilde{L}_{1}^{-1} \otimes \tilde{L}_{2}^{-1}\right)=0$,

- $h^{1}\left(\Theta_{\tilde{\mathbb{P}}^{3}}\left(\log \tilde{D}_{1}\right) \otimes \tilde{L}_{2}^{-1}\right)=0$.

Proof. We follow the idea of [7, section 5]. We have the exact sequence

$$
0 \longrightarrow \Theta_{\tilde{\mathbb{P}}^{3}}\left(\log \left(\tilde{D}_{1}\right)\right) \otimes \tilde{L}_{2}^{-1} \longrightarrow \Theta_{\tilde{\mathbb{P}}^{3}} \otimes \tilde{L}_{2}^{-1} \longrightarrow N_{\tilde{D}_{1} \mid \tilde{\mathbb{P}}^{3}} \otimes \tilde{L}_{2}^{-1} \longrightarrow 0 .
$$

We prove now some lemmas.

Lemma 1.19. The following equality holds.

$$
h^{0}\left(N_{\tilde{D}_{1} \mid \tilde{\mathbb{P}}_{3}} \otimes \tilde{L}_{2}^{-1}\right)=0
$$

Proof of Lemma. The divisor $\left.\left(\tilde{D}_{1} \otimes \tilde{L}_{2}^{-1}\right)\right|_{\tilde{D}_{1}}$ is not effective as its index of intersection with the proper transforms of the rays of the cone $D_{1}$ is negative.

Lemma 1.20. The following equalities hold.

$$
h^{1}\left(\Theta_{\tilde{\mathbb{P}} 3} \otimes \tilde{L}_{2}^{-1}\right)=h^{1}\left(\tilde{\mathbb{P}}^{3}, \Theta_{\tilde{\mathbb{P}} 3} \otimes \tilde{L}_{1}^{-1} \otimes \tilde{L}_{2}^{-1}\right)=0
$$

Proof of Lemma. We use the Leray spectral sequences associated to the blowings up. The number $h^{1}\left(\tilde{\mathbb{P}}^{3}, \Theta_{\tilde{\mathbb{P}}^{3}} \otimes \tilde{L}_{1}^{-1} \otimes \tilde{L}_{2}^{-1}\right)$ is computed explicitly in [7, section 5] as $\tilde{\mathbb{P}}^{3}$ is a partial step to resolving the octic $D_{1}+D_{2}$. To compute $h^{1}\left(\Theta_{\tilde{\mathbb{P}} 3} \otimes \tilde{L}_{2}^{-1}\right)$ we use the same argument as in the cited paper. We proceed by recursion. Let $\sigma: \tilde{P} \rightarrow P$ be a single blow up from the sequence leading from $\mathbb{P}^{3}$ to $\tilde{\mathbb{P}^{3}}$. As $D_{2}$ does not pass through any center of the blowings up $\tilde{L}_{2}$ can be replaced in each step of the recursion by $\sigma^{*}\left(L_{2}\right)$. We use the equality

$$
h^{1}\left(\Theta_{\tilde{P}} \otimes \sigma^{*}\left(L_{2}\right)^{-1}\right)=h^{1}\left(\Omega_{\tilde{P}}^{2} \otimes K_{P}^{\vee} \otimes \sigma^{*}\left(L_{2}\right)^{-1}\right)=h^{2}\left(\Omega_{\tilde{P}}^{1} \otimes K_{P} \otimes \sigma^{*}\left(L_{2}\right)\right)
$$

and the exact sequence

$$
\begin{aligned}
0 \longrightarrow \sigma^{*}\left(\Omega_{P}^{1} \otimes K_{P} \otimes L_{2}\right) & \otimes \mathcal{O}_{\tilde{P}}(k E) \longrightarrow \Omega_{\tilde{P}}^{1} \otimes K_{P} \otimes \sigma^{*}\left(L_{2}\right) \longrightarrow \\
\longrightarrow & \Omega_{E / C}^{1} \otimes \mathcal{O}_{E}(-k) \otimes \sigma^{*}\left(K_{P} \otimes L_{2}\right) \longrightarrow 0,
\end{aligned}
$$

where $C$ is the blown up set, $k=\operatorname{codim}_{P}(C)-1$, and $E$ is the exceptional divisor. We have $k>0$. From the projection formula we deduce

$$
\sigma_{*}\left(\Omega_{\tilde{P}}^{1} \otimes K_{P} \otimes \sigma^{*}\left(L_{2}\right)\right)=\Omega_{P}^{1} \otimes K_{P} \otimes L_{2} \otimes \sigma_{*}\left(\mathcal{O}_{\tilde{P}}(k E)\right)=\Omega_{P}^{1} \otimes K_{P} \otimes L_{2} .
$$

From the derived exact sequence we obtain $R^{i} \sigma_{*}\left(\Omega_{\tilde{P}}^{1} \otimes K_{P} \otimes \sigma^{*}\left(L_{2}\right)\right)=0$ for $i \geq 1$, since $R^{i} \sigma_{*}\left(\Omega_{E / C}^{1}(-k)=0\right.$ for $i \geq 0$ and $R^{i} \sigma_{*}\left(\mathcal{O}_{\tilde{P}}(k E)\right)=0$ for $i \geq 1$. Using 
the Leray spectral sequence and the recursion we get at the end $h^{1}\left(\Theta_{\tilde{\mathbb{P}} 3} \otimes \tilde{L}_{2}^{-1}\right)=$ $h^{1}\left(\Theta_{\mathbb{P}^{3}} \otimes L_{2}^{-1}\right)=0$.

The exact sequence 1.3 together with the lemmas proves the proposition.

The second component $h^{1}\left(\hat{Z}, \Theta_{\hat{Z}}\left(\log \hat{D}_{2}\right)\right)$ of the sum can be interpreted as the dimension of the space of deformations of $\hat{D}_{2}$ contained in deformations of $\hat{Z}$. It is thus the space of equisingular deformations of $\tilde{D}_{2}$ in deformations of $\tilde{Z}$. Let us first find a geometric interpretation of the space of all small deformations of $\tilde{Z}$. To do this we compute as above

$$
h^{1}\left(\tilde{Z}, \Theta_{\tilde{Z}}\right)=h^{1}\left(\tilde{\mathbb{P}}^{3}, \Theta_{\tilde{\mathbb{P}}^{3}} \otimes \tilde{L}_{1}^{-1}\right)+h^{1}\left(\Theta_{\tilde{\mathbb{P}}^{3}}\left(\log D_{1}\right)\right) .
$$

Now, $h^{1}\left(\Theta_{\tilde{\mathbb{P}}^{3}}\left(\log D_{1}\right)\right)$ represents the space of equisingular deformations of $D_{1}$ in $\mathbb{P}^{3}$. These deformations are also cones as they are of degree 4 and admit a fourfold point. Moreover, they correspond to equisingular deformations of the base quartic of $D_{1}$ in $\mathbb{P}^{2}$. The dimension of the space of transverse deformations is given by

$$
h^{1}\left(\tilde{\mathbb{P}}^{3}, \Theta_{\tilde{\mathbb{P}}^{3}} \otimes \tilde{L}_{1}^{-1}\right)=\text { The number of blowings up of double curves. }
$$

The above two spaces generate together the space of all quartic cones in $\mathbb{P}^{3}$. Hence, every deformation of $\tilde{Z}$ is a resolution of the double cover $Z$ of $\mathbb{P}^{3}$ branched over a quartic cone. Next, observe that a deformation of $\tilde{D}_{1}$ in a deformation of $\tilde{Z}$ corresponds to a deformation of its projection onto $Z$. The latter is a deformation of a complete intersection of two quartics in $\mathbb{P}(1,1,1,2)$ hence is also an intersection of this type. Thus equisingular deformations of $\tilde{D}_{1}$ in deformations of $\tilde{Z}$ are a subset of deformations of a pair of cones in $\mathbb{P}^{3}$ with fixed vertices. It remains to observe that the equisingularity condition imposes on the deformed cones the same tangency and incidence conditions as in the original cones.

Remark 1.21. All above works also if we allow any reduced fibers. The only difference is that during the resolution of the branch locus of the second covering we obtain singularities that are not nodes. These are some double and triple points admitting small crepant resolutions. As these are only double and triple points they do not affect the space of transversal deformations. The rest remains unchanged.

Remark 1.22. We can use the same argument to compute deformations of the fiber product of general elliptic surfaces with section. Indeed, most non reduced fibers correspond to triple curves on the cones. We treat the triple curves in the following way. We blow them up and add the exceptional divisor to the proper transform of the branch locus on the blowing up to obtain an even divisor. The rest remains unchanged.

\section{KumMer FIBRATIONS}

In this section we study a fiberwise Kummer construction for a product of rational elliptic surfaces with section.

Let $X$ be the fiber product (possibly singular) of two rational elliptic surfaces $S_{1}$ and $S_{2}$ with section. Let $a=\left(a_{1}, a_{2}\right): \mathbb{P}^{1} \longrightarrow X$ be any section on $X$. Then $a_{1}$ and $a_{2}$ are sections of $S_{1}$ and $S_{2}$ respectively. Let us consider the involution $i: X \longrightarrow X$ such that on each smooth fiber it is of the form $i\left(x_{1}, x_{2}\right)=\left(a_{1}-x_{1}, a_{2}-x_{2}\right)$. Observe that $i$ is well defined as we have a group structure on each fiber. Let $Y$ be the quotient of $X$ by the involution $i$. 
Proposition 2.1. In the above setting $Y$ admits a resolution being a Calabi-Yau three fold (not necessarily projective).

Before the proof of the proposition let us consider more closely the involution $i$. Let $i_{1}$ and $i_{2}$ denote the involutions on $S_{1}$ and $S_{2}$ such that $i=\left(i_{1}, i_{2}\right)$. We study the possible actions of these involutions on a singular fiber $F$ of $S_{1}$ or $S_{2}$ respectively.

Lemma 2.2. Let $S$ be a rational elliptic surface with chosen 0 section and such that $S$ admits only reduced fibers. Let $j$ be an involution of the form $x \mapsto b-x$, where $b$ is a section of $S$. Let $F$ be a singular fiber of $S$. Then exactly one of the following possibilities occurs.

(1) The fiber $F$ is of type $I_{1}$ and $j$ acts on $F$ by symmetry.

(2) The fiber $F$ is of type $I_{2 k}$. Then we have one of the following cases.

(a) The involution $j$ has two fixed points (these are then two opposite nodes) and interchanges pairs of components of $F$.

(b) The involution $j$ acts on two opposite components of $F$ by symmetry, interchanging respective remaining components.

(3) The fiber $F$ is of type $I_{2 k+1}$ for $k \geq 1$. Then $j$ acts on one of the components of $F$ by symmetry with fixed points outside singularities of $F$ and interchanges the respective pairs of remaining components.

(4) The fiber $F$ is of type II. Then $j$ fixes the singular point of $F$ and admit one more fixed point.

(5) The fiber $F$ is of type III. Then we have two possibilities:

(a) The involution $j$ has only one fixed point and interchanges the two components of $F$.

(b) The involution $j$ acts on both components fixing their intersection point and one more point on each fiber.

(6) The fiber $F$ is of type $I V$. Then the involution $j$ fixes the triple point, interchanges two of the components of the fiber and acts on the third component, admitting an additional fixed point.

Proof of lemma. Following [16] for each fiber $F$ of $S$ there is an induced group structure on $F^{\sharp}$, the set of smooth points of the fiber. The involution $j$ is of the form $x \mapsto b-x$ on each fiber including the singular ones. Hence $j$ acts also in this way on the group $F^{\sharp} / F^{0}$ of connected components of $F^{\sharp}$. The latter group for a reduced fiber is isomorphic to $\mathbb{Z} / n \mathbb{Z}$. On this group we have three possibilities for an involution of the form $[x] \mapsto[b]-[x]$.

- The number $n$ is odd. Then the involution has one fixed point (i.e. $j$ acts on exactly one of the component of the singular fiber)

- The number $n$ is even but $[b]$ is odd. Then the involution has no fixed points (i.e. $j$ interchanges pairs of components of the singular fiber).

- The numbers $n$ and $[b]$ are both even. Then the involution has two fixed points (i.e. $j$ acts on exactly two of the component of the singular fiber).

Moreover, the structure group on $F^{0}$, the component of the zero section, is multiplicative for $F=I_{n}$ and additive for the remaining fibers. This means that an involution fixing a component of the fiber $I_{n}$ admits two fixed points on the smooth part of this fiber. In the same way an involution fixing a component of a fiber different from $I_{n}$ admits one fixed point on the smooth part of this fiber. Together we obtain all possibilities described in the assertion of the lemma. 
The above lemma implies immediately the following

Corollary 2.3. Locally around a fixed point $P$ of $j$ we have the following possibilities.

(1) The fixed point $P$ is a smooth point of the fiber on which it is lying.

(2) The fiber admits a node in $P$ and the local analytic components of this node are interchanged by the involution $j$.

(3) The fiber is of type III singular at $P$ and the components of this fiber are interchanged by the involution $j$.

(4) The fiber is of type III. The point of intersection is fixed but the involution acts on both components separately.

(5) The fiber is of type $I V$ and all three of its components pass through P. Two of them are interchanged by the involution.

Corollary 2.4. We can perform a local analytic change of coordinates around $P$ in such a way that the fibration is preserved and the surface $S$ is given in $\mathbb{C}^{3}$ with coordinates $(x, t, u)$ by the equation:

(1) $u^{2}=x$ and the fibration is given by $t$,

(2) $u^{2}=x^{2}-t$ and the fibration is given by $t$,

(3) $u^{2}=x^{4}-t$ and the fibration is given by $t$,

(4) $u^{2}=x-t$ and the fibration is given by $x t$,

(5) $u^{2}=x^{2}-t$ and the fibration is given by $x t$.

Where the involution is given by $u \mapsto-u$.

Proof. We need only to observe that each of the cases from the corollary [2.3] is represented locally by one of these equations.

Let us come back now to the proposition.

Proof of Proposition 2.1. By the adjunction formula for a double covering, as $Y$ is a double cover of a Calabi-Yau three fold branched over a set of codimension 2, the canonical divisor $K_{Y}$ is trivial. We need only to find a crepant resolution of the singularities. Outside fixed points of the involution the singularities are locally isomorphic to the corresponding singularities of $X$, hence admit a crepant resolution. Moreover, from the Corollary 2.4 around the fixed points of the involution $i$ we have the following possibilities for the local equation of the quotient variety.
(a) $u^{2}=x y$
(b) $u^{2}=\left(x^{2}-t\right) y$
(c) $u^{2}=\left(x^{4}-t\right) y$
(d) $u^{2}=(x-t) y$
(e) $u^{2}=\left(x^{2}-t\right)\left(y^{2}-t\right)$
(f) $u^{2}=\left(x^{4}-t\right)\left(y^{2}-t\right)$
(g) $u^{2}=(x-t)\left(y^{2}-x t\right)$
(h) $u^{2}=\left(x^{4}-t\right)\left(y^{4}-t\right)$
(i) $u^{2}=\left(x^{2}-t\right)\left(y^{2}-x t\right)$
(j) $u^{2}=(x-t)\left(y^{4}-x t\right)$
(k) $u^{2}=(x-t)(y-z), x t=y z$

The first step in the resolution of the quotient variety is the blowing up of the branch curve. Observe that the first four possibilities are transversal $A_{1}$ singularities. It means that the above blow up gives their crepant resolution. In all remaining cases 
except case (k) after blowing up the singular curve we get isolated singularities with small resolution.

(e) We get two nodes.

(f) We get two singularities of type $A_{3}$.

(g) We get two singularities of type $D_{4}$.

(h) We get two singularities given in suitable local coordinate systems by the equation $x^{4}-y^{4}=u^{2}-t^{2}$.

(i) We get a singularity of type $A_{5}$.

(j) We get a singularity given in a suitable local coordinate system by the equation $x^{4}-y^{4}=u^{2}-t^{2}$.

In the last case after blowing up the singular curve we obtain a new singular curve that we next blow up again. As the blown up curves were double curves the obtained resolution is also crepant.

From now on we assume moreover that $S_{1}$ and $S_{2}$ admit only semi-stable singular fibers. This means that in the above list of local equations of singularities of the Kummer fibration we allow only types (a),(b) and (e).

Proposition 2.5. If $X$ has only semi-stable fibers and no fibers of type $I_{1} \times I_{n}$, then the three fold $Y$ admits a projective Calabi-Yau resolution.

Proof. Observe first that outside the fixed locus $X^{i}$ of the involution the proposition is trivial as the double cover $X \backslash X^{i} \longrightarrow Y \backslash X^{i}$ is étale.

It remains to see that blowing up separately the strict transforms of all components of all fibers we can resolve the nodes that appeared on the partial resolution (after blowing up the double curves) of $Y$. Here the only case we need to check more closely is what happens over a singular point of the fifth type from the proof of Proposition 2.1. After blowing up the branch curve (locally it is defined by the equations $x^{2}-t=y^{2}-t=u=0$ ) the two components of the fiber $t=0$ (given locally by equations $t=0, u= \pm x y$ ) remain smooth and pass through the obtained nodes. The fact that these are indeed two global components follows from the assumption that fibers are semi-stable and no fiber is of type $I_{1} \times I_{n}$.

2.1. Hodge numbers. We compute Hodge numbers of the constructed projective varieties. Let us start by computing their Euler characteristics. Let us denote the branch curve of the involution $i$ by $C$. From the above construction we have

$$
\chi(\hat{Y})=\frac{\chi(X)-\chi(C)}{2}+2 \chi(C)+2 o+b,
$$

where $o$ denotes the number of singular points of $X$ fixed by the involution and $\mathrm{b}$ denotes the half of the number of the remaining nodes of $X$. The number $\chi(C)$ can be computed from the number of fixed points on each fiber.

Lemma 2.6. We have the following equality:

$$
\chi(C)=16\left(2-\sharp\left(A_{1} \cup A_{2}\right)\right)+\sum_{t \in A_{1} \cup A_{2}} a_{t} a_{t}^{\prime},
$$

where $a_{t}$ and $a_{t}^{\prime}$ are the number of fixed points of the involutions $i_{1}$ and $i_{2}$ on fibers lying over $t$ of the respective surfaces $S_{1}$ and $S_{2}$.

Proof. The proof is straightforward as $C$ admits a projection onto $\mathbb{P}^{1}$ with generic fiber consisting of 16 points. Special fibers appear only on singular fibers of the fibration. 
Remark 2.7. Depending on the type of fibers the numbers $a_{t}$ and $a_{t}^{\prime}$ take only one of the three values $\{2,3,4\}$. Moreover, $a_{t}=3$ if and only if the fiber $F_{t}$ of $S_{1}$ is of type $I_{n}$ for $\mathrm{n}$ odd.

Remark 2.8. The Euler characteristic of $\hat{Y}$ depends on the involution $i$ i.e. for some Calabi-Yau fiber products we can produce Kummer three folds with different Hodge numbers.

The remark is illustrated by the following example.

Example 2.9. Let $S_{1}$ and $S_{2}$ be double covers of $\mathbb{P}^{2}$ branched over generic quartic curves. Let their elliptic fibrations be chosen in such a way that $S_{1}$ has exactly one fiber of type $I_{2}$ (one of the lines defining a fiber is double tangent to the quartic), all remaining singular fibers of $S_{1}$ are of type $I_{1}$, and all singular fibers of $S_{2}$ are of type $I_{1}$. Let the 0 sections of both surfaces be given by the pre-image of the points defining the fibrations. We denote by $i$ the involution defined by the zero section of the product and by $j=\left(j_{1}, j_{2}\right)$ the involution induced by the coverings defining each surface. The involution $j=\left(j_{1}, j_{2}\right)$ is also an involution of type $\left(a_{1}-x_{1}, a_{2}-x_{2}\right)$ because both $j_{1}$ and $j_{2}$ have four fixed points on the generic fiber of $S_{1}$ and $S_{2}$. The sections $a_{1}$ (resp. $a_{2}$ ) is the section for which the preimages of the branch quartic defining $S_{1}$ (resp. $S_{2}$ ) by the double covering represent the four-section given by the equation $2 x_{1}=a_{1}$ (resp. $2 x_{2}=a_{2}$ ) in the group structure of each smooth fiber of $S_{1}$. As we have chosen everything to be generic, the product $S_{1} \times_{\mathbb{P}^{1}} S_{2}$ is already smooth, hence $o=b=0$. Both involutions on each fiber of the fibration different from the fiber $I_{2} \times I_{0}$ have the same number of fixed points. However, on this fiber they differ ( $i$ has 4 fixed points but $j$ only two) giving different $\chi(C)$ and consequently different $\chi(\hat{Y})$. More precisely $\chi\left(\hat{Y}^{i}\right)=-74$ and $\chi\left(\hat{Y}^{j}\right)=-96$.

Let us now concentrate on computing the deformations of $\hat{Y}$. To do so we will need some more results concerning elliptic surfaces with involution.

Lemma 2.10. Let $S$ be a rational elliptic surface with a chosen 0 section. Let $b$ be any section of $S$. Let $j$ be the involution given in each smooth fiber by $x \mapsto b-x$. Then at least one of the following two possibilities hold:

- The surface $S$ is birational to the double cover of $\mathbb{P}^{2}$ branched in a quartic curve with ADE singularities. This birational equivalence can be chosen in such a way that the fibration of $S$ is given by proper transforms of lines passing through a point on $\mathbb{P}^{2}$ and that the involution $j$ corresponds to the involution of the covering.

- The surface is birational to the double cover of a quadric cone in $\mathbb{P}^{3}$ (i.e. $\mathbb{P}(1,1,2))$ branched in the vertex and the intersection of this cone with a smooth cubic not passing through the vertex. The birational equivalence can be taken in such a way that the fibration is given by the proper transforms of the rays of the cone and the involution $j$ corresponds to the involution of the covering.

Proof. Observe that taking the quotient of the surface $S$ by the involution $j$ we obtain a ruled surface $R$ over $\mathbb{P}^{1}$. This ruled surface is either $\mathbb{P}^{1} \times \mathbb{P}^{1}$ or it admits a section $s$ which is an exceptional curve.

Let us first consider the second possibility. Choose $s$ and blow down all components of the fibers disjoint from it. In this way we obtain a minimal ruled surface 
with section $s$. Note that each component of the pullback of $s$ by the covering is a -1 curve and a section on the elliptic surface. Now taking into account the position of $s$ with respect to the branch divisor we can compute its self-intersection number. We have three possibilities:

- The section $s$ is contained in the branch locus of the quotient by the involution. Then the pullback of $s$ by the covering is a double -1 curve. The self-intersection number of $s$ is then equal to -2 . Hence $s$ is the -2 curve on the ruled surface $\mathbb{F}_{2}$.

- The section $s$ is disjoint from the branch locus of the quotient by the involution. Then its preimage by the covering has two disjoint components with self-intersection -1. The self-intersection number of $s$ is then equal to -1 . Hence $s$ is the -1 curve on the ruled surface $\mathbb{F}_{1}$.

- The section $s$ cuts the branch locus of the quotient by the involution. Then the preimage of $s$ by the covering is either irreducibe with even selfintersection or has two intersecting components. In both cases $s$ has to have non-negative self-intersection hence cannot be an exceptional curve.

After blowing down $s$ the assertion follows.

The remaining possibility is that the ruled surface $R$ is $\mathbb{P}^{1} \times \mathbb{P}^{1}$. In this case we can perform an elementary transformation with center at a tangency point of some fiber with the branch locus on $\mathbb{P}^{1} \times \mathbb{P}^{1}$ to obtain $\mathbb{F}_{1}$. We then need only to blow down the exceptional section of $\mathbb{F}_{1}$ to obtain (a) from the assertion.

Remark 2.11 . The above lemma is very similar to Lemma 1.12. The only difference is that we take care not only of the surface and the fibration but also of the chosen involution. This additional requirement forces us to consider one more possibility. However in the above proof if at least one fiber of the elliptic surface has more than two components, then we have a choice of the section $s$ and can choose it not to be contained in the ramification divisor. This means that the only cases where the elliptic surface with a chosen involution is not birational to the double cover of $\mathbb{P}^{2}$ branched over a quartic curve with its natural involution is when all fibers of the surface are irreducible and $b$ is the 0 -section.

Now we can describe the Kummer fibration $\hat{Y}$.

Corollary 2.12. The three fold $\hat{Y}$ is birational to one of the following:

(a) The resolution of the double cover of $\mathbb{P}^{3}$ branched over the sum $D$ of two possibly reducible quartic cones.

(b) The resolution of the double cover of $\mathbb{P}(1,1,1,2)$ branched over the sum of two possibly reducible weighted cones of degrees 4 and 6 .

(c) The resolution of the double cover of $\mathbb{P}(1,1,2,2)$ branched over the sum of two possibly reducible weighted cones of degrees 6 and 6 .

Moreover, the Kummer fibration is given by proper transforms of planes passing through the vertices of both cones.

Remark 2.13. Using Lemma 1.12 for every fiber product $X$ we can always construct an involution $i$ on $X$ such that the corresponding Kummer fibration $\hat{Y}$ satisfies case (a).

With regard to the above remark we consider only the case (a) of Corollary 2.12 We comment later also the remaining cases, however we omit details. 
Let us assume that $\hat{Y}$ is projective and that the fixed points set of the involution $i$ does not contain the zero section. In this case $\hat{Y}$ is birational to the resolution of the double covering of $\mathbb{P}^{3}$ branched along an octic surface $D$, which is the sum of two quartic cones $Q_{1}$ and $Q_{2}$. From this point of view the natural smooth model to study is described by the following construction. We blow up $\mathbb{P}^{3}$ consecutively in all the fourfold points and the double curves of the branch locus. Next, we take a small resolution of all obtained nodes. This smooth model will be denoted by $\tilde{Y}$.

Remark 2.14. We have two different natural constructions of smooth models of introduced Kummer fibrations. The first one was described in the proof of Proposition 2.1 the second is described above. In the generic case (e.g. the fiber product of two elliptic surfaces with singular fibers only of type $I_{1}$ and no common singular fibers) these two resolutions differ by the composition of two flops. More precisely in this case the first model arises from the second by blowing up each of the two components of the proper transform of the line passing through the vertices of the cones and contracting the second rulings of the obtained in this way quadrics.

In general the situation is more complicated. The described resolutions differ more when the elliptic surfaces admit common singular fibers which are fixed by the involution, i.e. when the two cones $Q_{1}$ and $Q_{2}$ are singular along some lines that intersect each other.

Example 2.15. To illustrate the second part of the above remark let us consider the following two elliptic surfaces. Let $S_{1}$ (resp. $S_{2}$ ) be the resolution of the double covering of $\mathbb{P}^{2}$ with coordinates $(x, z, t)$ (resp. $\left.(y, z, t)\right)$ blown up in the point $(1,0,0)$, branched over the proper transforms of the nodal quartic $q_{1}=\{x(x+z)(x-t-z)(x+t-z)=0\}\left(\right.$ resp. $q_{2}=\{y(y+z)(y-t-7 z)(y+$ $t+5 z)=0\})$. Let their fibrations be given by the projection onto $(z, t)$. Observe that in this setting $S_{1}$ (resp. $S_{2}$ ) has singular fibers only of type $I_{2}$ in the points $\{(1,-2),(1,-1),(1,0),(1,1),(1,2),(0,1)\}$ (resp. $\{(1,-8),(1,-7),(1,-6)$, $(1,-5),(1,-4),(0,1)\})$. This means that teir fiber product admits one fiber of type $I_{2} \times I_{2}$ in the point $(0,1)$ and all remaining fibers are of type $F \times I_{0}$. The product is equipped with a natural involution coming from the involution of the covering on each surface. The Kummer fibration corresponding to this involuton is birational to the double covering of $\mathbb{P}^{3}$ with coordinates $(x, y, z, t)$ branched over the sum of two quartic cones $Q_{1}=\{x(x+z)(x-t-z)(x+t-z)=0\}$ and $Q_{2}=\{y(y+z)(y-t-7 z)(y+t+5 z)=0\}$. Let us denote the vertices of the cones by $V_{1}=(0,1,0,0), V_{2}=(1,0,0,0)$ and the remaining fourfold point of the octic surface $D=Q_{1} \cup Q_{2}$ by $P=(0,0,0,1)$. The variety $\tilde{Y}$ introduced in the discussion above is obtained by blowing up $\mathbb{P}^{3}$ in the points $P, V_{1}$ and $V_{2}$, next blowing up the proper transforms of the lines $P V_{1}$ and $P V_{2}$ and the remainng doble lines of the cones, then blowing up the proper transform of the intersection $Q_{1} \cap Q_{2}$, and finally taking the double covering of the obtained variety branched over the proper tranform of $D$. By straightforward computation in local coordinates we can prove that the resolution $\hat{Y}$ described in the proof of Theorem 2.1 can be obtained from the resolution $\tilde{Y}$ by a sequence of four disjoint flops. Beside flopping the two components of the preimage by the covering of the proper transform of the line $V_{1} V_{2}$ we need to flop the two components of the preimage by the covering of the proper transform of the line of intersection of the exceptional divisor lying over $P$ with the proper transform of the plane $P V_{1} V_{2}$. 
Let us remind that the hodge numbers of Calabi-Yau threefolds are birational invariants. Hence to compute the deformations of the manifold $\hat{Y}$ we can use results of [7] applied to $\tilde{Y}$. The dimension of the space of transversal deformations of $\tilde{Y}$ is equal to the geometric genus of the sum of all double curves of the branch locus. As the double curves of each cone are rational curves the only curve we need to deal with is the curve of intersection of the cones denoted by $C_{I}$.

Remark 2.16. The curve $C_{I}$ is birational to the branch curve $C$ of the involution on the fiber product. We already know how to compute the Euler characteristic of the curve $C$ (see Lemma 2.6 ). Moreover, we know that $C$ is smooth outside the nodes in the points of the fifth type from the proof of Proposition 2.1. That is we know the Euler characteristic of the resolution of $C$.

This allows us to compute the geometric genus of $C_{I}$ provided we also know the number of its components. Unfortunately this last number cannot be deduced from the types of fibers of the fiber product and the type of involutions induced on each of these fibers. This is shown by the following example.

Example 2.17. Let $S_{1}$ be the double cover of $\mathbb{P}^{2}$ branched over the sum of a line and a nodal cubic cutting transversely. Let $S_{1}^{\prime}$ be the double cover of $\mathbb{P}^{2}$ branched over a quartic with three nodes. Let $S_{2}$ be the double cover of $\mathbb{P}^{2}$ branched over the sum of four generic lines. Let the fibration in each case be given by preimages of lines passing through a generic point of the plane. Then $S_{1} \times_{\mathbb{P}^{1}} S_{2}$ has the same types of fibers as $S_{1}^{\prime} \times_{\mathbb{P} 1} S_{2}$ and the same type of involution (the type is given by the number of fixed points) on each fiber. However the number of components of the branch locus on the two three folds differ. In consequence the dimensions of transversal deformations also differ. We will see later that the space of equisingular deformations are equal in both cases. Hence it is not enough to know the types of fibers of the fibration and the involution on each fiber to compute the Hodge numbers of a Kummer surface.

Remark 2.18. We can find a bound to the number of components by studying the components of both cones. A lower bound is given by the product of the number of components of both cones. Moreover, with few exception this lower bound is in fact the actual number.

By the methods of 7 it remains to compute the equisingular deformation of the surface $D$. As we have assumed, $D$ is the sum of two quartic cones $Q_{1}$ and $Q_{2}$.

Proposition 2.19. The equisingular deformations of the branch locus $D$ correspond to a subset of deformations of the fiber product $\hat{X}$ consisting of those deformations that induce by Theorem 1.15 equisingular deformations of each of the quartic cones $Q_{1}$ and $Q_{2}$.

Proof. We need first to prove that an equisingular deformation of $D$ induces a deformation of the fiber product $\hat{X}$.

To do this we observe that an equisingular deformation of the sum of two quartic cones in $\mathbb{P}^{3}$ is also a sum of two quartic cones. This follows from the fact that the curve of intersection of the two cones is a complete intersection $(4,4)$ and hence has to be preserved in the deformation as a double curve which is also a complete intersection of type $(4,4)$. As we know that the fourfold points are preserved the claim follows. 
Next, we prove that the $I_{n} \times I_{m}$ fibers are preserved in the equisingular deformations of $D$. We proceed as follows, observe that a fiber of this type arises in one of the following ways.

- The plane corresponding to the fiber is tangent to both cones.

- The plane corresponding to the fiber is tangent to one of the cones and contains some singular lines (one or two) of the second cone.

- The plane corresponding to the fiber contains some singular lines of both cones.

Let us now observe that a double line is deformed into double lines and a fourfold point has to be deformed into a fourfold point. Thus the configuration of double lines is deformed with the same incidence relations. Observe moreover that the intersection points of lines tangent to both cones are also preserved in the deformation as they induce two nodes on the blow up of $\mathbb{P}^{3}$ in the curve of intersection of the cones. The same concerns the intersection points of a double line with a tangency line. This proves the claim as all fibers of type $I_{n} \times I_{m}$ are defined by the incidence relations between double and tangency lines of the cones.

Together this gives an inclusion of the set of all equisingular deformations of $D$ to the set of equisingular deformations of the fiber product. The inclusion in the opposite direction follows from the fact that we can perform a simultaneous resolution of singularities in the family of all equisingular deformations of the cones.

Remark 2.20. Although general formulas describing the deformation space of a studied Kummer fibrations are rather difficult to write, all above computations are very easy for explicit examples.

Remark 2.21. The above reasoning as it stands works only for Kummer fibrations satisfying the case (a) from Corollary [2.12. However the method of [7] should also work in the remaining cases provided we prove that the surrounding weighted projective spaces admit rigid resolutions. This is not hard as we have a description of such spaces as a hypersurface and a complete intersection respectively.

Remark 2.22. We have one more approach to deal with the cases (b) and (c) from Corollary 2.12 We can use Remark 2.11 to translate almost all Kummer fibrations (except the products of a surfaces with only $I_{1}$ singularities) into resolutions of double covers of $\mathbb{P}^{3}$ branched over a sum of quartic cones. We need only to allow the cones to pass through each other vertices. To this more general picture we can also use results of [7, the only difference is that we have fivefold points, which are resolved in a more complicated but also allowed way.

Remark 2.23. As earlier we can generalize the above results to non projective Kummer fibrations.

We know how to compute the deformations and the Euler characteristic of the Kummer fibration $\hat{Y}$, hence we can easily compute the rank of its Picard group.

Example 2.24. Let us take the most general fiber product. That is a smooth variety $X$ which is a product of two elliptic surfaces with only $I_{1}$ fibers (the singular fibers of the product are only of type $I_{1} \times I_{0}$ ). The suitable Kummer fibration is then the resolution $\hat{Y}$ of the double cover of $\mathbb{P}^{3}$ branched over the sum of two generic 
quartic cones. We have the following table of invariants.

\begin{tabular}{c|c|c|c} 
& $\chi$ & $h^{1,2}$ & $h^{1,1}$ \\
\hline$X$ & 0 & 19 & 19 \\
$\hat{Y}$ & -96 & 52 & 4
\end{tabular}

\section{REFERENCES}

[1] W. Barth, C. Peters, A. Van de Ven, Compact complex surfaces, Heidelberg, Berlin, New York, Springer 1984.

[2] S. Cynk, Defect of a nodal hypersurface, Manuscripta math. 104 (2001), 325-331.

[3] S. Cynk, Cyclic coverings of Fano threefolds, Ann. Polon. Math. 80 (2003), 117-124.

[4] S. Cynk, Cohomologies of a double covering of a nonsingular algebraic 3-folds, Math. Z. 240(2) (2002) 731-743.

[5] S. Cynk, Double coverings of octic arrangements with isolated singularities, Adv. Theor. Math. Phys. 3 (1999), 217-225.

[6] S. Cynk, C. Meyer, Geometry and Arithmetic of certain Double Octic Calabi-Yau Manifolds, Canad. Math. Bull. 48(2) (2005) 180-194.

[7] S. Cynk, D. van Straten, Infinitesimal deformations of smooth algebraic varieties, Math. Nachr. 279(7) (2006) 716-726.

[8] G. Faltings, Endlichkeitssätze für abelsche Varietäten über Zahlkörpern, Inv. Math. 73 (1983), 349366.

[9] R. Friedman, Simultaneous resolution of threefold double points, Math. Ann. 274(4) (1986) 671-689.

[10] G.-M. Greuel, G. Pfister, H. Schönemann. Singular 2.0. A Computer Algebra System for Polynomial Computations. Centre for Computer Algebra, University of Kaiserslautern (2001). http://www.singular.uni-kl.de.

[11] M. Gross, S. Popescu, Calabi-Yau Threefolds and Moduli of Abelian Surfaces I, Compositio Math.127(2) (2001), 16

[12] G. Heckman, E. Looijenga, The moduli space of rational elliptic surfaces, Algebraic geometry 2000, Azumino (Hotaka), 185-248, Adv. Stud. Pure Math., 36, Math. Soc. Japan, Tokyo, 2002.

[13] S.Herfurtner, Elliptic surfaces with four singular fibres, Math. Ann 291 (1991) 319-342.

[14] B. Hunt, A bound on the Euler number for certain Calabi-Yau 3-folds, J. Reine Angew. Math. 411 (1990) 137-170

[15] A.R. Iano-Fletcher, Working with weighted complete intersections, in A. Corti and M. Reid, editors, Explicit birational geometry of 3-folds, LMS Lecture Notes Series 281, CUP, Cambridge, 2000, 101-173.

[16] V. A. Iskovskikh, I. R. Shafarevich, Algebraic surfaces. Algebraic geometry, II, 127-262, Encyclopaedia Math. Sci., 35, Springer, Berlin, 1996.

[17] J. Kollár, Flops, Nagoya Math. J. 113 (1989), 15-36.

[18] J. Kollár, and S. Mori, Classification of Three-dimensional Flips, J. Amer. Math. Soc. 5 (1992) 533-703.

[19] J. Kollár, and S. Mori, Birational geometry of algebraic varieties, Cambridge University Press 134 (1998)

[20] H.-W. Lin, On crepant resolution of some hypersurface singularities and a criterion for UFD, Trans. Amer. Math. Soc. 354(5) (2002), 1861-1868 (electronic).

[21] R. Miranda, The basic theory of elliptic surfaces, Notes of lecture, ETS Editrice Pisa.

[22] R. Miranda, Persson's list of singular fibers for a rational elliptic surface, Math. Z. 205(2) (1990), 191-211.

[23] R. Miranda, U. Persson, On Extremal Rational Elliptic Surfaces, Math. Z. 193(1986), $537-558$

[24] R. Miranda; U. Persson, Torsion groups of elliptic surfaces, Compositio Math. 72(3) (1989), 249-267.

[25] K. Oguiso, On the finiteness of fiber-space structures on a Calabi-Yau threefold, J. Math. Sci. 106(5) (2001), 3320-3335.

[26] U. Persson, Configurations of Kodaira fibers on rational elliptic surfaces, Math. Z. 205(1) (1990), 1-47. 
[27] S. Rams, Defect of Hodge number of hypersurfaces, Habilitationschrift Erlangen 2006.

[28] Z. Ran, Lifting of cohomology and unobstructedness of certain holomorphic maps, Bull. Amer. Math. Soc. (N.S.) 26(1) (1992) 113-117.

[29] Z. Ran, Hodge theory and deformations of maps, Compositio Math. 97(3) (1995) 309-328.

[30] M. Reid, Canonical 3-folds, Geometrie Algebrique Angers, (A. Beauville, ed.), Sijtho K+Noordhoof, (1980) 273310.

[31] M. Reid, Chapters on algebraic surfaces, in Complex algebraic varieties, J. Kollár Ed., IAS/Park City lecture notes series (1993 volume),AMS, 1997, 1-154.

[32] M. Reid Young person's guide to canonical singularities, in Algebraic Geometry, Bowdoin 1985, ed. S. Bloch, Proc. of Symposia in Pure Math. 46, A.M.S. (1987), vol. 1, 345-414.

[33] C. Schoen, On Fiber Products of Rational Elliptic Surfaces with Section, Math. Z. 197 (1988), 177-199.

[34] C. Schoen, On the computation of the cycle class map, Ann. Sci. École Norm. Sup. 28(1) (1995) $1-50$

[35] E. Sernesi, Deformations of algebraic schemes, Grundlehren der Mathematischen Wissenschaften [Fundamental Principles of Mathematical Sciences], 334. Springer-Verlag, Berlin, 2006.

[36] P.M.H. Wilson, Calabi-Yau manifolds with large Picard number, Invent. Math. 98(1) (1989), 139-155.

[37] P.M.H. Wilson, The Kaehler Cone on Calabi-Yau Threefolds, Invent. Math. 107 (1992) 561-583.

[38] P.M.H. Wilson, Erratum to The Kaehler Cone on Calabi-Yau Threefolds, Invent. Math. 114 (1993) 231-233.

[39] P.M.H. Wilson, Towards birational classification of algebraic varieties, Bull. London Math. Soc. 19 (1987) 1-48.

[40] P.M.H. Wilson, Elliptic ruled surfaces on Calabi-Yau threefolds, Math. Proc. Cambridge Philos. Soc. 112 (1992), 45-52.

[41] P.M.H. Wilson, The existence of elliptic fiber space structures on Calabi-Yau threefolds, Math. Ann. 300 (1994), 693-703

[42] P.M.H. Wilson, The existence of elliptic fiber space structures on Calabi-Yau threefolds, II, Math. Proc. Camb. Phil. Soc. 123(2) (1998), 259-262.

Grzegorz Kapustka

Jagiellonian University

ul. Reymonta 4

30-059 Kraków

Poland

email:

Grzegorz.Kapustka@im.uj.edu.pl
Michał Kapustka

Jagiellonian University

ul. Reymonta 4

30-059 Kraków

Poland

email:

Michal.Kapustka@im.uj.edu.pl 